\title{
Analysis of patients undergoing urological intervention amid the COVID-19: experience from the pandemic hospital
}

\author{
Mustafa Soytas ${ }^{1}$ - Mustafa Yucel Boz ${ }^{1} \cdot$ Vahit Guzelburc $^{1} \cdot$ Gokhan Calik $^{1} \cdot$ Mehmet Cagri Kactan ${ }^{1} \cdot$ Rahim Horuz $^{1}$. \\ ${\text { Ziya } \text { Akbulut }^{1} \cdot \text { Selami Albayrak }}^{1}$
}

Received: 26 May 2020 / Accepted: 20 June 2020 / Published online: 24 June 2020

(c) Springer Nature B.V. 2020

\begin{abstract}
Purpose It is reported that surgical procedures performed during the COVID-19 pandemic are accompanied by high complications and risks. In this study, the urological interventions applied with appropriate infrastructure and protocols during the pandemic in the pandemic hospital that is carrying out the COVID-19 struggle are analyzed.

Methods Urological interventions were reviewed in the 5-week period between March 11 and April 16. The distribution of outpatient and interventional procedures was determined by weeks concurrently along with the COVID-19 patient workload, and data in the country, subgroups were further analyzed. Patients intervened were divided into four groups as Emergency, High, Intermediate, and Low Priority cases according to the EAU recommendations. The COVID-19-related findings were recorded; staff and patient effects were reported.

Results Of the 160 interventions, 65 were minimally invasive or open surgical intervention, 95 were non-surgical outpatient intervention, and the outpatient admission was 777. According to the priority level, 33 cases had emergency and high priority, 32 intermediate and low priority. COVID-19 quarantine and follow-up were performed at least 1 week in 22 (33.8\%) operated patients at the last week, $43(66.2 \%)$ patients who were operated in the previous 4 weeks followed up at least 2 weeks. No postoperative complications were encountered in any patient due to COVID-19 during the postoperative period.

Conclusion In the COVID-19 pandemic, precautions, isolation, and algorithms are required to avoid disruption in the intervention and follow-up of urology patients; priority urological interventions should not be disrupted in the presence of necessary experience and infrastructure.
\end{abstract}

Keywords Coronavirus · COVID-19 $\cdot$ Elective case $\cdot$ Emergency case $\cdot$ Priority case $\cdot$ SARS-CoV-2 $\cdot$ Urology

Mustafa Soytas

drmustafasoytas@gmail.com

Mustafa Yucel Boz

myboz@medipol.edu.tr

Vahit Guzelburc

vahitguzelburc@hotmail.com

Gokhan Calik

drgokhan80@hotmail.com

Mehmet Cagri Kactan

cagrikactan@gmail.com

Rahim Horuz

rhoruz@medipol.edu.tr

Ziya Akbulut

zakbulut@medipol.edu.tr

Selami Albayrak

salbayrak@medipol.edu.tr

1 Department of Urology, Faculty of Medicine, Istanbul Medipol University, 34200 Istanbul, Turkey

\section{Introduction}

COVID-19 (SARS-CoV-2) was recorded as the third coronavirus outbreak of the twenty-first century after SARS-CoV-1 and MERS [1]. The World Health Organization (WHO) accepted coronavirus disease 2019 (COVID-19) as a global pandemic and declared as an emergency on March 11, 2020 [2]. The first case was reported in Turkey on the same date by the Ministry of National Health [3]. Even though various prediction models and forecasts about when the process will end or the second wave of infection will be introduced, uncertainty still remains [4].

We do not know how to maintain social life and daily urology practice in all this uncertainty. With this unprecedented condition, the knowledge regarding COVID-19 and urological practice has begun to accumulate rapidly. However, until now, most of this accumulated knowledge 
has come from expert opinions, survey results, and some limited case series reports and research. The knowledge here is dispersed, confused, and controversial.

One of the forefront reports of the literature Lei et al. has reported intensive care unit (ICU) need in $44.1 \%$ and the mortality was $20.5 \%$ of patients who underwent surgery during the pandemic [5]. The urological associations suggested that 'Low priority' cases (which can be postponed for the longest time) can be postponed for 6 months in their guidelines [6]. However, we do not know what the situation will be after 6 months or can not predict how to manage postponed patients.

In our study, we aimed to present the precautions which have taken in our clinic during the COVID-19 pandemic process and COVID-19-related follow-up, results of patient examinations, and interventions (Low Priority, Intermediate Priority, High Priority, and Emergency).

\section{Methods}

Urological interventions were reviewed in the 5-week period between March 11 when the first case of COVID19 was reported in our country, and April 16. Data collection was decided on April 16, and patients after that date were excluded. Inpatient visits are prohibited from the beginning of the pandemic. While emergency cases were included at the beginning of the pandemic, priority cases started to be included in the following days. In addition to the surgical consent form, the COVID-19 information and consent form prepared by us which added as an appendix was also signed by the patients. The distribution of the number of outpatient and interventional procedures were determined by weeks. Simultaneously, hospital COVID-19 patient burden and COVID-19 patient data in the country were evaluated. Age, gender, and comorbid diseases were determined. While international recommendations have not yet emerged in the early stages of the pandemic, the approach to cases was determined according to internal guidelines. National Health Ministry guidelines and EAU, AUA, and ASCO suggestions were taken into consideration in the following period. The COVID-19 algorithm of our clinic was applied to all patients (Fig. 1). In all patients, the COVID-19-PCR test was performed after the outbreak announcement, and chest computed tomography (CT) was performed in suspicious patients. Patients who underwent surgery and non-surgical procedures were divided into 4 groups as Low Priority, Intermediate Priority, High Priority, and Emergency according to the EAU recommendations. The COVID-19-compatible symptoms, complaints, mortality, and morbidity conditions of the patients were recorded by contacting the patients.

\section{Results}

In the 5-week period since 11 March when the first case was reported, the number of COVID-19 cases of the hospital has been reported as 5217 , the number of cases in the city as 53,186 , and the number of cases in the country as 90,980. Our hospital consists of 2 blocks. The first block is used for COVID-19 patients and the other one where we work, patients without COVID are treated and followed. 1 of the $3 \mathrm{CT}$ devices in the hospital is only available for patients with COVID-19 suspicion. While 2 of the 3 ICUs in our hospital were reserved for COVID-19 patients, 1 was used for COVID-19 (-) and postoperative patients. At the start of the process, half of the team waited at home, considering that urology staff might be needed. In this process, seven urologists and three residents worked in the clinic, and two specialists from the team were infected with COVID-19, returned to the clinic after treatment, and became an immune plasma donor to eight ICU patients (MS, RH). All physicians were assigned to the patient examination in the COVID-19 building, which was once a month, and on the 5th day after the task, COVID-19-PCR tests were performed, and the negative ones continued their daily urology practice. COVID-19 quarantine and follow-up were performed at least 1 week for $22(33.8 \%)$ patients who were operated in the last week, and at least 2 weeks for $43(66.2 \%)$ patients who were operated in the previous 4 weeks.

During the 5-week period, the number of operations performed in our clinic is 65 , the number of non-operative interventions is 95 and the number of outpatient examinations is 777 . In the 5 -week period prior to the pandemic, the number of surgeries was 127 , the number of non-operative interventions was 264, and the outpatient examinations were 1384. During the pandemic period, the number of operations and outpatient examinations decreased by approximately half, compared to the pandemic period; while, the number of non-operative interventions decreased by 1 in 3 . The mean age of the operated patients was 47 and 45 were male $(69.2 \%)$ and 20 were female $(30.8 \%)$ (Table 1$)$. When the surgeries were evaluated, 18 were classified as Emergency, 15 were High Priority, 4 were Intermediate Priority, and 28 were Low Priority (Table 2). While RIRS can be considered as low priority case under normal conditions, bacteremia developed in 1 patient due to proximal ureter stones, and a D-J stent was tried to place but failed. Flexible ureterorenoscopy (F-URS) was performed for the stone in the proximal ureter of the patient who could not be placed the guidewire, and after fragmentation and removal of the obstruction with F-URS, a D-J stent was inserted and the patient was left to the second session. Four ureterorenoscopy patients 


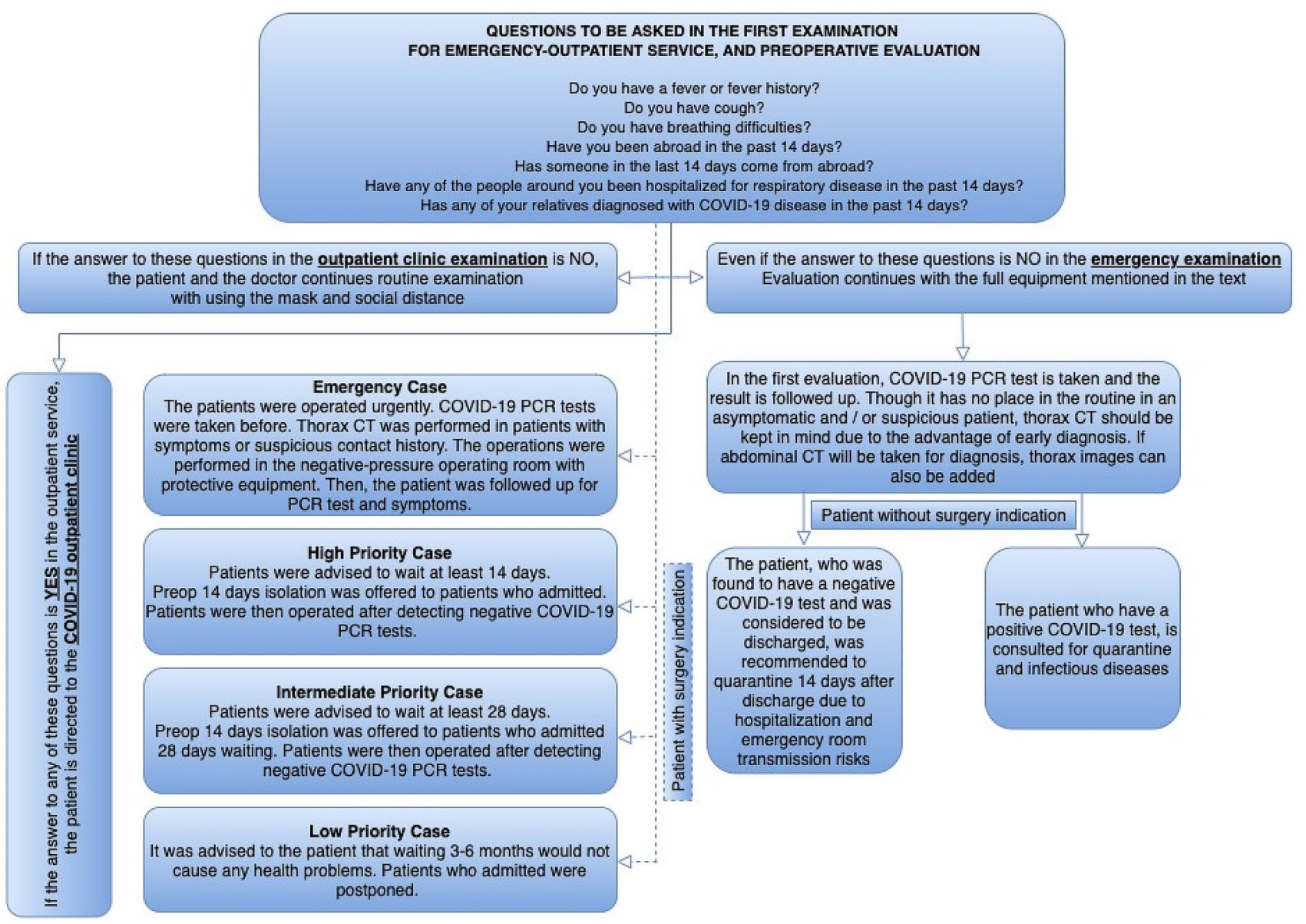

Fig. 1 COVID-19 patient evaluation and approach algorithm

Table 1 Demographics and comorbidities

\begin{tabular}{lll}
\hline & Total (N: 65) & No./\% \\
\hline Age, mean, year \pm std & $47 \pm 14.7$ & \\
Sex & & \\
Male & $45 / 69.2 \%$ \\
Female & $20 / 30.8 \%$ \\
Comorbidity & \\
Malignancy & $14 / 21.5 \%$ \\
Chronic kidney disease (CKD) & $7 / 10.7 \%$ \\
Hypertension & $5 / 7.6 \%$ \\
Diabetes & $4 / 6.1 \%$ \\
Cardiovascular disease & $4 / 6.1 \%$ \\
Chronic obstructive pulmonary disease & $2 / 3.1 \%$ \\
Cerebrospinal disease & $1 / 1.6 \%$ \\
\hline
\end{tabular}

were evaluated as high priority cases, and 1 of them presented with severe pain that did not respond to analgesics and 3 of them with elevated creatinine. As non-surgical intervention the number of urethral catheters was 20 , uroflowmetry was 64, urodynamics was 3, ESWL was 4, BCG was 4 . The total number of outpatient patients was 777. The outpatient, surgical and non-surgical procedures have been determined by weeks, and although the numbers have decreased towards the middle of the process, and tended to increase in the last 2 weeks (Fig. 2). Although no complication due to COVID-19 was observed in any patient during the postoperative period, 2 patients who underwent radical cystectomy, and radical nephrectomy and vena cava thrombectomy were followed up at the ICU for 1 day postoperatively.

\section{Discussion}

The SARS-CoV-1 epidemic infected more than 8000 people in 29 countries between 2002 and 2004, caused more than 800 deaths [7, 8]. Although it is an epidemic and causes fewer cases and deaths than the COVID-19 pandemic, the spread of SARS-CoV-1 over 2 years cause pessimism about how long the pandemic process will continue. Today, we 
Table 2 Surgical, non-surgical interventions and COVID-19 follow-up

\begin{tabular}{|c|c|c|c|c|c|c|}
\hline & Emergency & High priority & $\begin{array}{l}\text { Intermediate } \\
\text { priority }\end{array}$ & Low priority & Total & $\begin{array}{l}\text { COVID-19 testing in } \\
7-14 \text { days follow-up }\end{array}$ \\
\hline \multicolumn{7}{|l|}{ Surgeries } \\
\hline \multicolumn{7}{|l|}{ Radical nephrectomy } \\
\hline Open & & & 2 & 2 & 4 & Negative \\
\hline Laparoscopic & & & 1 & & 1 & Negative \\
\hline Radical cystectomy + IC & & & 1 & & 1 & Negative \\
\hline Robotic radical prostatectomy & & & & 1 & 1 & Negative \\
\hline Radical orchiectomy & 1 & & & & 1 & Negative \\
\hline TUR-bladder & 1 & 10 & & & 11 & Negative \\
\hline TRUS prostate biopsy & & & & 2 & 2 & Negative \\
\hline Transvesical prostatectomy & & & & 1 & 1 & Negative \\
\hline RIRS & & 1 & & 8 & 9 & Negative \\
\hline Ureterorenoscopy & 7 & 4 & & 4 & 15 & Negative \\
\hline Double-J removal & & & & 6 & 6 & Negative \\
\hline Double-J stenting & 4 & & & & 4 & Negative \\
\hline Testicular exploration & 1 & & & & 1 & Negative \\
\hline Nephrostomy insertion & 4 & & & & 4 & Negative \\
\hline Internal urethrotomy & & & & 1 & 1 & Negative \\
\hline Botox application & & & & 1 & 1 & Negative \\
\hline Penile fracture repair & & & & 1 & 1 & Negative \\
\hline Sacral neuromodulation & & & & 1 & 1 & Negative \\
\hline Total & 18 & 15 & 4 & 28 & 65 & \\
\hline \multicolumn{7}{|l|}{ Non-surgical procedures } \\
\hline Urodynamics & & & & 3 & 3 & Negative \\
\hline Uroflowmetry & & & & 64 & 64 & Negative \\
\hline Urethral catheterization & 20 & & & & 20 & Negative \\
\hline ESWL & & & & 4 & 4 & Negative \\
\hline BCG-session & & & & 4 & 4 & Negative \\
\hline Total & 20 & & & 75 & 95 & \\
\hline
\end{tabular}

$I C$ ileal conduit, RIRS retrograde intrarenal surgery, TUR transurethral resection, TRUS transrectal ultrasonography, ESWL extracorporeal shock wave lithotripsy, $B C G$ Bacillus Calmette-Guérin

face 2397,216 cases and 162,956 deaths in the COVID-19 pandemic [9].

In our country, the pandemic is taken into account seriously. At the beginning of the pandemic, there was a fear that the health system could collapse in government officials and the public, and there was concern that many deaths would occur. The science committee chaired by our health minister carries out the pandemic process. The pandemic guide prepared by the scientific board and updated almost every day is used throughout the process [3]. Apart from those applying to the hospital, people with suspicious contamination in their environment were screened by the Ministry of Health filiation teams with the COVID-19 PCR test. There is no restriction or difficulty in using the COVID-19 PCR test kit. In the preoperative evaluation of the patients, COVID19 PCR results were concluded within 6-12 h. As of today, the process is carried out successfully and no problems have been experienced in terms of the health system.

We do not really know which stage of the COVID-19 pandemic we are in. Although the whole world hopes that it will end in a few months and life will return to normal, we cannot predict the genetic behavior of the virus or whether there will be two or more waves of infection [4]. In some countries, cases and deaths started to decrease but in another country or continent the pandemic is just beginning to spread [10]. All this uncertainty forces us to adapt to the conditions using the resources we have. Because other diseases do not have to wait for the pandemic and the annual mortality of many diseases, especially malignancies, is higher than the mortality of COVID-19 [11]. The general consensus shows that even 'Low priority' cases can be delayed for 6 months [6]. 
Fig. 2 Patient and procedure distribution in COVID-19 pandemic by weeks
PATIENT AND PROCEDURE DISTRIBUTION

IN COVID-19 PANDEMIC BY WEEKS

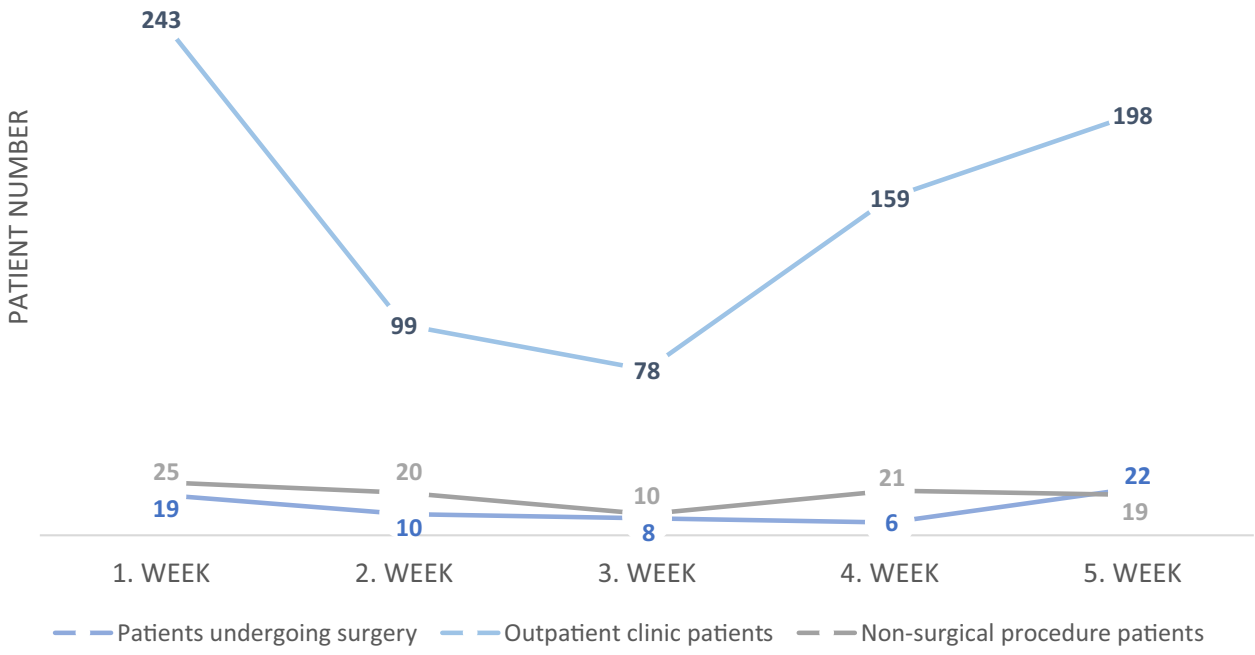

To adapt to this process, urology associations and many other surgical branches have published various algorithm and suggestion guidelines $[6,12,13]$. Unfortunately, expert opinions and suggestions are given due to the lack of evidence-based data when these guidelines are examined. These suggestions are presented by taking into account the mortality and morbidity rates of the diseases over time. At this point, we have created our own internal suggestion guide by our clinic sub-branches and started to implement it [14].

Very high rates of ICU requirement and mortality were reported in a study which presenting mortality and morbidity during the COVID-19 incubation period [5]. In this study, 34 patients were evaluated and intensive care was required in $15(44.1 \%)$ of these patients, and approximately half of them died $(20.5 \%)$. The disadvantage of these patients is being in the incubation period and this seems to be the cause of high mortality. In our study, the process was managed according to our algorithm, and 14-day incubation period was waited to prevent infected patients and then the patients were operated. Emergency operated patients were accepted as COVID19-PCR (+) and operated in negative pressure operation rooms; then, COVID-19-PCR results were followed. Even if the COVID-19-PCR results are negative, they are warned to avoid leaving home and maintain social distance for at least 14 days due to an average of 5-day incubation period and possible hospital contamination [15]. Routine COVID19 test was not applied to the patients after the operation. Patients were warned about COVID-19-related symptoms and were questioned by telephone.

The city where the study is conducted has the highest number of cases in the country with more than 50,000 cases according to today's data [16]. We are one of the pandemic hospitals with the highest number of patients, and 5217 patients were examined during this 5 -week period. Despite all these risk factors and patient burden, COVID-19 transmission was not detected in any of our patients due to the algorithms and principles we applied. COVID quarantine and follow-up were performed at least 1 week for 22 patients $(33.8 \%)$ which operated in the last week, and at least 2 weeks for 43 patients $(66.2 \%)$ who were operated in the previous 4 weeks. In addition, CO2-dependent COVID-19 transmission risk caused anxiety during our laparoscopic-robotic operations, as in gynecological laparoscopic surgery, but this problem was tried to be reduced by the smoke evacuation system [17].

In a study examining the comorbidities of hospitalized patients for COVID-19, the rate of hypertension, diabetes and cardiovascular disease stands out [18]. The most common comorbid diseases in our study were malignancies $(21.5 \%)$, chronic kidney failure $(10.7 \%)$, hypertension (7.6\%), diabetes (6.1\%), respectively. We tended to postpone in comorbid patients and we were more careful in terms of transmission risk and mortality rate if we had to operate these patients.

\section{Conclusion}

As before the pandemic, emergency patients were treated without any delay after appropriate precautions were taken. All priority patients were informed about postponement and treated according to the clinical COVID-19 algorithm. 
COVID-19 infection was not encountered in any patient who underwent intervention. If the necessary precautions, isolations, and algorithms are followed, the COVID-19 process will be overcome with minimal damage, and urological interventions can be done safely in the pandemic. Priority urological interventions should not be disrupted in the presence of necessary experience, equipment, and infrastructure.

Author contributions MS: project development, data collection, analysis and interpretation, manuscript writing/editing. MYB, VG, GC, MCK, RH, ZA and SA: data collection, analysis and interpretation.

\section{Compliance with ethical standards}

Conflict of interest The authors declare that they have no conflict of interest.

Ethical standards All procedures performed in studies involving human participants were in accordance with the ethical standards of the institutional and/or national research committee and with the 1964 Helsinki Declaration and its later amendments or comparable ethical standards.

Informed consent This is a retrospective review. For this type of study, formal consent is not required.

\section{References}

1. Guarner J (2020) Three emerging coronaviruses in two decades the story of SARS, MERS, and now COVID-19. Am J Clin Pathol. https://doi.org/10.1093/ajcp/aqaa029

2. WHO (2020) WHO Timeline-COVID-19. Publishing whoweb. https://www.who.int/news-room/detail/08-04-2020-who-timel ine-covid-19. Accessed 16 June 2020

3. Bakanligi S (2020) New COVID-19. Publishing saglikweb. https ://covid19bilgi.saglik.gov.tr/depo/rehberler/COVID-19_Rehbe ri.pdf. Accessed 16 June 2020

4. Yi Y, Lagniton PNP, Ye S et al (2020) COVID-19: what has been learned and to be learned about the novel coronavirus disease. Int J Biol Sci. https://doi.org/10.7150/ijbs.45134

5. Lei S, Jiang F, Su W et al (2020) Clinical characteristics and outcomes of patients undergoing surgeries during the incubation period of COVID-19 infection. EClinicalMedicine 000:100331. https://doi.org/10.1016/j.eclinm.2020.100331
6. EAU (2020) COVID-19 Recommendations. Publishing eauweb. https://uroweb.org/guideline/covid-19-recommendations/Acces sed 16 June 2020

7. WHO (2004) Summary of probable SARS cases with onset of illness from 1 November. 2002 to 31 July 2003. Publishing whoweb. https://www.who.int/csr/sars/country/table2004_04_21/ en/. Accessed 16 June 2020

8. WHO (2004) China's latest SARS outbreak has been contained, but biosafety concerns remain-Update 7. Publishing whoweb. https://www.who.int/csr/don/2004_05_18a/en/. Accessed 16 June 2020

9. WHO (2020) Coronavirus disease 2019 (COVID-19) Situation Report-78. Publishing whoweb. https://www.who.int/docs/defau lt-source/coronaviruse/situation-reports/20200407-sitrep-78-covid -19.pdf?sfvrsn=bc43e1b_2. Accessed 16 June 2020

10. WHO (2020) Coronavirus disease 2019 (COVID-19) Situation Report-91. Publishing whoweb. https://www.who.int/docs/defau 1t-source/coronaviruse/situation-reports/20200420-sitrep-91-covid -19.pdf?sfvrsn=fcf0670b_4. Accessed 16 June 2020

11. Siegel RL, Miller KD, Jemal A (2020) Cancer statistics, 2020. CA Cancer J Clin. https://doi.org/10.3322/caac. 21590

12. FACS (2020) COVID-19 guidelines for triage of urology patients. Publishing facsweb. https://www.facs.org/covid-19/clinical-guida nce/elective-case/urology. Accessed 16 June 2020

13. AUA (2020) COVID-19 Info Center. Publishing AUAweb. https ://www.auanet.org/covid-19-info-center/covid-19-info-center. Accessed 16 June 2020

14. Istanbul Medipol University Hospital (2020) COVID-19 (SARS-COV-2) PANDEMISII ÜROLOJİ HASTA YAKLAŞIM REHBERİ. Publishing medipolweb. https://www.medipol.com. $\mathrm{tr} /$ medium/document-file-104.vsf. Accessed 16 June 2020

15. Stahel PF (2020) How to risk-stratify elective surgery during the COVID-19 pandemic? Patient Saf Surg. https://doi.org/10.1186/ s13037-020-00235-9

16. Bakanligi S (2020). New COVID-19. Publishing saglikweb. https ://covid19bilgi.saglik.gov.tr/tr/. Accessed 16 June 2020

17. Mallick R, Odejinmi F, Clark TJ (2020) Covid 19 pandemic and gynaecological laparoscopic surgery: knowns and unknowns. Facts Views Vis ObGyn 12:3

18. Wang D, Hu B, Hu C et al (2020) Clinical characteristics of 138 hospitalized patients with 2019 novel coronavirus-infected pneumonia in Wuhan, China. JAMA J Am Med Assoc. https://doi. org/10.1001/jama.2020.1585

Publisher's Note Springer Nature remains neutral with regard to jurisdictional claims in published maps and institutional affiliations. 